\title{
ON THE MARKOV TRANSITION KERNELS FOR FIRST PASSAGE PERCOLATION ON THE LADDER
}

\author{
ECKHARD SCHLEMM, ${ }^{*}$ Technische Universität München
}

\begin{abstract}
We consider the first passage percolation problem on the random graph with vertex set $\mathbb{N} \times\{0,1\}$, edges joining vertices at a Euclidean distance equal to unity, and independent exponential edge weights. We provide a central limit theorem for the first passage times $l_{n}$ between the vertices $(0,0)$ and $(n, 0)$, thus extending earlier results about the almost-sure convergence of $l_{n} / n$ as $n \rightarrow \infty$. We use generating function techniques to compute the $n$-step transition kernels of a closely related Markov chain which can be used to explicitly calculate the asymptotic variance in the central limit theorem.
\end{abstract}

Keywords: Central limit theorem; first passage percolation; generating function; Markov chain; transition kernel

2010 Mathematics Subject Classification: Primary 60K35; 60J05

Secondary $33 \mathrm{C} 90$

\section{Introduction}

The subject of first passage percolation, introduced in [11] in 1965, is the study of shortest paths in random graphs. Let $G=(V, E)$ be a graph with vertex set $V$ and unoriented edges $E \subset V^{2}$, and assume that there is a weight function $w: E \rightarrow \mathbb{R}_{+}$. For vertices $u, v \in V$, a path joining $u$ and $v$ in $G$ is a sequence of vertices $\boldsymbol{p}_{u \rightarrow v}=\left\{u=p_{0}, p_{1}, \ldots, p_{n-1}, p_{n}=v\right\}$ such that $\left(p_{v}, p_{v+1}\right) \in E$ for $0 \leq v<n$. The weight $w\left(\boldsymbol{p}_{u \rightarrow v}\right)$ of such a path is defined as the sum of the weights of the comprising edges,

$$
w\left(\boldsymbol{p}_{u \rightarrow v}\right):=\sum_{\nu=0}^{n-1} w\left(\left(p_{\nu}, p_{\nu+1}\right)\right) .
$$

The first passage time between the vertices $u$ and $v$ is denoted by $d_{G}(u, v)$ and defined as $d_{G}(u, v):=\inf \{w(\boldsymbol{p}), \boldsymbol{p}$ a path joining $u$ and $v$ in $G\}$.

First passage percolation can be considered a model for the spread of a fluid through a random porous medium; it differs from ordinary percolation theory in that it puts special emphasis on the dynamical aspect of how long it takes for certain points in the medium to be reached by the fluid. Important applications include the spread of infectious diseases (see [4]) and the analysis of electrical networks (see [10]). Recently, there has also been an increased interest in first passage percolation on graphs where not only the edge weights, but the edge structure itself is random. These models, including the Gilbert and Erdős-Rényi random graphs, were investigated in [5], [18], and [19], and found to be useful approximations to the Internet as well as telecommunication networks.

Received 22 July 2010; revision received 17 December 2010.

* Postal address: TUM Institute for Advanced Study \& Zentrum Mathematik, Technische Universität München, Boltzmannstraße 3, 85748 Garching bei München, Germany. Email address: schlemm@ma.tum.de 
Usually, however, the underlying graph is taken to be $\mathbb{Z}^{2}$ and the edge weights are independent random variables with some common distribution P; see, e.g. [17] and the references therein. Interesting mathematical questions that arise in this context concern the asymptotic properties of the sets $B_{t}:=\left\{u \in \mathbb{Z}^{2}: d_{\mathbb{Z}^{2}}(0, u) \leq t\right\}$ [12], [16], and the limiting behaviour of $d_{\mathbb{Z}^{2}}((0,0),(n, 0)) / n$. The latter is known to converge, under weak assumptions on $\mathrm{P}$, to a deterministic constant, called the first passage percolation rate; the computation of this constant has proved to be a very difficult problem and has not yet been accomplished even for the simplest choices of $\mathrm{P}$ (see [9, p. 1937]). An exception to this is the case when the underlying graph $G$ is essentially one-dimensional. (See [8], [14], and [15].)

In this paper we consider the first passage percolation problem on the ladder $G$, a particular essentially one-dimensional graph, for which the first passage percolation rate is known [14], [15]. We extend the existing results about the almost-sure convergence of $d_{G}((0,0),(n, 0)) / n$ as $n \rightarrow \infty$ by providing a central limit theorem as well as giving a complete description of the $n$-step transition kernels of a closely related Markov chain. Our results can be used to explicitly compute the asymptotic variance in the central limit theorem. They are also the basis for the quantitative analysis of any other statistic related to the first passage percolation in this model. In particular, knowledge of the higher-order transition kernels is the starting point for the computation of the distribution of the rungs which are part of the shortest path. The ladder model is worth studying because it is one of the very few situations where a complete explicit description of the finite-time behaviour of the first passage percolation times can be given.

The structure of the paper is as follows. In Section 2 we describe the model and state our results. Section 3, which contains the proofs, is divided into three subsections: Subsection 3.1 is devoted to the central limit theorem, in Subsection 3.2 we present some explicit evaluations of infinite sums which are needed in Subsection 3.3, where we prove the main theorem about the transition kernels. We conclude the paper with a brief discussion.

We use the notation $\delta_{p, q}$ for the Kronecker delta, and $\Theta_{p, q}$ as well as $\tilde{\Theta}_{p, q}$ for versions of the discretized Heaviside step function:

$$
\delta_{p, q}:=\left\{\begin{array}{ll}
1 & \text { if } p=q, \\
0 & \text { otherwise }
\end{array} \quad \Theta_{p, q}:=\left\{\begin{array}{ll}
1 & \text { if } p \geq q, \\
0 & \text { otherwise }
\end{array} \quad \tilde{\Theta}_{p, q}:= \begin{cases}1 & \text { if } p \leq q, \\
0 & \text { otherwise }\end{cases}\right.\right.
$$

The symbol $(k)^{!}$stands for $k !(k+1)$ !. We denote by $\mathbb{R}$ the real numbers and by $\mathbb{Z}$ the integers. Subscripts ' + ' and ' - ' indicate restrictions to the positive and negative elements of a set, respectively. The symbol $\gamma$ stands for the Euler-Mascheroni constant and E denotes the expectation.

\section{First passage percolation on the ladder}

In this paper we further investigate a first passage percolation model which has been considered before in [14] and also in [15]. We denote by $G_{n}$ the graph with vertex set $V_{n}=\{0, \ldots, n\} \times\{0,1\}$ and edges

$$
\begin{aligned}
& \chi_{n}=\{((i, 0),(i+1,0)): 0 \leq i<n\}, \\
& \mathcal{y}_{n}=\{((i, 1),(i+1,1)): 0 \leq i<n\}, \\
& \mathcal{Z}_{n}=\{((i, 0),(i, 1)): 0 \leq i \leq n\} .
\end{aligned}
$$

The edge weights are independent, exponentially distributed random variables which are labelled in the obvious way as $X_{i}, Y_{i}$, and $Z_{i}$; see also Figure 1 . By time scaling, it is no 


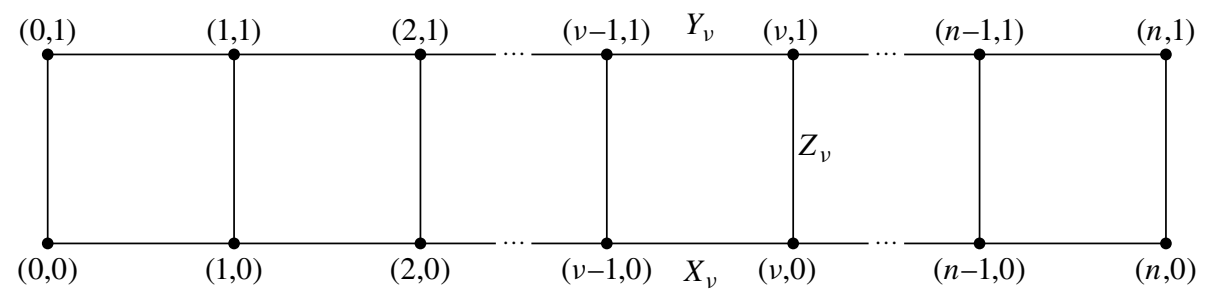

Figure 1: The ladder graph $G_{n}$. The edge weights $X_{v}, Y_{\nu}$, and $Z_{v}$ are independent exponential random variables.

restriction to assume that the edge weights have mean 1 . We further denote by $l_{n}$ the length of the shortest path from $(0,0)$ to $(n, 0)$ in the graph $G_{n}$, by $l_{n}^{\prime}$ the length of the shortest path from $(0,0)$ to $(n, 1)$, and by $\Delta_{n}$ the difference between the two, i.e. $\Delta_{n}=l_{n}^{\prime}-l_{n}$. It has been shown in [15] and also, by a different method, in [14] that $\lim _{n \rightarrow \infty} l_{n} / n$ almost surely exists and is equal to the constant $\chi=\frac{3}{2}-J_{1}(2) / 2 J_{2}(2)$, where $J_{v}$ are Bessel functions of the first kind. (See Definition 1 below or [1, Chapter 9] for a comprehensive review of Bessel functions.) This constant is called the first passage percolation rate for our model. The method employed in [15] to obtain this result built on [8] and consisted in showing that there exists an ergodic $\left(\mathbb{R} \times \mathbb{R}_{+}^{3}\right.$ )-valued Markov chain $M=\left(M_{n}\right)_{n \geq 0}$ with stationary distribution $\tilde{\pi}$ and a function $f: \mathbb{R} \times \mathbb{R}_{+}^{3} \rightarrow \mathbb{R}$ such that

$$
\chi=\mathrm{E} f\left(M_{\infty}\right)=\int_{\mathbb{R} \times \mathbb{R}_{+}^{3}} f(m) \tilde{\pi}(\mathrm{d} m) .
$$

Explicitly,

$$
M_{n}=\left(\Delta_{n}, X_{n+1}, Y_{n+1}, Z_{n+1}\right) \quad \text { and } \quad f:(r, x, y, z) \mapsto \min \{r+y+z, x\} .
$$

Throughout, we write $m=(r, x, y, z)$ for some element of the state space $\mathbb{R} \times \mathbb{R}_{+}^{3}$. In order to better understand the first passage percolation problem on the ladder, it is important to know the higher-order transition kernels $\tilde{K}^{n}:\left(\mathbb{R} \times \mathbb{R}_{+}^{3}\right) \times\left(\mathbb{R} \times \mathbb{R}_{+}^{3}\right) \rightarrow \mathbb{R}_{+}$of the Markov chain $M$. They completely determine the dynamics of the model and are defined as

$$
\tilde{K}^{n}\left(m^{\prime}, m\right) \mathrm{d} m=\mathrm{P}\left(M_{n} \in \mathrm{d} m \mid M_{0}=m^{\prime}\right), \quad m, m^{\prime} \in \mathbb{R} \times \mathbb{R}_{+}^{3} .
$$

The first result shows that it is sufficient to control the transition kernels $K^{n}: \mathbb{R} \times \mathbb{R} \rightarrow \mathbb{R}_{+}$of the Markov chain $\Delta=\left(\Delta_{n}\right)_{n \geq 0}$, which are analogously defined as

$$
K^{n}\left(r^{\prime}, r\right) \mathrm{d} r=\mathrm{P}\left(\Delta_{n} \in \mathrm{d} r \mid \Delta_{0}=r^{\prime}\right), \quad r, r^{\prime} \in \mathbb{R} .
$$

For convenience, we define $K^{0}\left(r^{\prime}, r\right):=\delta_{r^{\prime}}(r)$, the Dirac distribution.

Proposition 1. For any $n \geq 1$, denote by $\tilde{K}^{n}$ the $n$-step transition kernel of $M$ defined in (1). Then

$$
\tilde{K}^{n}\left(m^{\prime}, m\right)=\mathrm{e}^{-(x+y+z)} K^{n-1}\left(\min \left\{r^{\prime}+y^{\prime}, x^{\prime}+z^{\prime}\right\}-\min \left\{r^{\prime}+y^{\prime}+z^{\prime}, x^{\prime}\right\}, r\right) .
$$

Moreover, the stationary distribution $\tilde{\pi}$ of $M$ is given by

$$
\tilde{\pi}(\mathrm{d} m)=\mathrm{e}^{-(x+y+z)} \mathrm{d}^{3}(x, y, z) \pi(\mathrm{d} r),
$$


where

$$
\pi(\mathrm{d} r)=\frac{1}{2 J_{2}(2)} \mathrm{e}^{-3|r| / 2} J_{1}\left(2 \mathrm{e}^{-|r| / 2}\right) \mathrm{d} r
$$

is the stationary distribution of $\Delta$.

Next, we state a central limit theorem for first passage percolation times on the ladder which was implicit in [15] and which was the motivation for the current paper. In [2] and [6] a central limit theorem was obtained for first passage times on fairly general one-dimensional graphs by a different method. The question of how to compute the asymptotic variance was, however, not addressed there. We denote by $\bar{f}$ the mean corrected function $f-\chi$.

Theorem 1. For any integer $n \geq 0$, let $l_{n}$ denote the first passage time between $(0,0)$ and $(n, 0)$ in the ladder graph $G_{n}$. Then there exists a positive constant $\sigma^{2}$ such that

$$
\frac{l_{n}-n \chi}{\sqrt{n}} \stackrel{\mathrm{D}}{=} N\left(0, \sigma^{2}\right)
$$

where $N\left(0, \sigma^{2}\right)$ is a normally distributed random variable with mean 0 and variance $\sigma^{2}$, and ' $=$ ' denotes convergence in distribution. Moreover,

$$
\sigma^{2}=\int_{\mathbb{R} \times \mathbb{R}_{+}^{3}} \bar{f}(m)^{2} \tilde{\pi}(\mathrm{d} m)+2 \sum_{n=1}^{\infty} \int_{\mathbb{R} \times \mathbb{R}_{+}^{3}} \bar{f}(m) P^{n} \bar{f}(m) \tilde{\pi}(\mathrm{d} m),
$$

where

$$
P^{n} \bar{f}(m)=\mathrm{E}\left[\bar{f}\left(M_{n}\right) \mid M_{0}=m\right]=\int_{\mathbb{R} \times \mathbb{R}_{+}^{3}} \bar{f}\left(m^{\prime}\right) \tilde{K}^{n}\left(m, m^{\prime}\right) \mathrm{d} m^{\prime} .
$$

Equation (5) shows that in order to evaluate the asymptotic variance of the first passage times, we must know the transition kernels $\tilde{K}^{n}$. In the next theorem we therefore explicitly describe the structure of the transition kernel $K^{n}$ and, thus, by Proposition 1 , the structure of $\tilde{K}^{n}$. To state the formulae in a compact way, we define the five functions

$$
\begin{aligned}
S^{1}(z) & =\frac{z-2 J_{2}(2 \sqrt{z})}{z}, \\
S^{2}(z) & =\frac{2(z-1)+2 J_{0}(2 \sqrt{z})}{z}, \\
G(z) & =-\frac{z^{3}}{4}\left[5-4 \gamma+2 \pi \frac{Y_{2}(2 \sqrt{z})}{J_{2}(2 \sqrt{z})}-2 \log z\right], \\
\alpha(z) & =\frac{z^{4}}{4 \sqrt{z} J_{2}(2 \sqrt{z})\left[\sqrt{z} J_{0}(2 \sqrt{z})+(z-1) J_{1}(2 \sqrt{z})\right]},
\end{aligned}
$$

and

$$
H(z)=\frac{z^{2}}{2(1-z)}\left[3 z-3+2 \pi Y_{0}(2 \sqrt{z})+J_{0}(2 \sqrt{z})(5-4 \gamma-2 \log z)\right] .
$$

The Bessel functions $J_{v}$ and $Y_{v}$ are defined in Definition 1 below and treated comprehensively in [1]. 
Theorem 2. The transition kernels $K^{n}$, defined in (2), satisfy $K^{n}\left(r^{\prime}, r\right)=K^{n}\left(-r^{\prime},-r\right)$. For $r \geq 0$, the values $K^{n}\left(r^{\prime}, r\right)$ are given by

$$
K^{n}\left(r^{\prime}, r\right)= \begin{cases}\sum_{p, q=0}^{n} a_{p, q}^{n} \mathrm{e}^{p r^{\prime}-(q+2) r}, & r^{\prime} \leq 0, \\ \frac{(-1)^{n-1} \mathrm{e}^{r^{\prime}-(n+1) r}}{(n-1)^{!}}+\sum_{p=0}^{n-2} \frac{(-1)^{n} r^{\prime} \mathrm{e}^{-p\left(r^{\prime}-r\right)-n r}}{(p)^{!}(n-p-2)^{!}} & \\ +\sum_{p, q=0}^{n} b_{p, q}^{n} \mathrm{e}^{-p r^{\prime}-(q+2) r}, & 0<r^{\prime} \leq r, \\ \frac{(-1)^{n-1} \mathrm{e}^{-(n-1) r^{\prime}-r}}{(n-1)^{!}}+\sum_{p=0}^{n-2} \frac{(-1)^{n} r \mathrm{e}^{-p\left(r^{\prime}-r\right)-n r}}{(p)^{!}(n-p-2) !} & \\ +\sum_{p, q=0}^{n} c_{p, q}^{n} \mathrm{e}^{-p r^{\prime}-(q+2) r}, & r^{\prime}>r,\end{cases}
$$

where the coefficients $a_{p, q}^{n}, b_{p, q}^{n}$, and $c_{p, q}^{n}$ are determined by their generating functions:

(i) the generating functions $A_{p, q}(z)=\sum_{n=1}^{\infty} a_{p, q}^{n} z^{n}, p, q \geq 0$, are given by

$$
\begin{aligned}
& A_{1, q}(z)=\frac{(-z)^{q}}{(q)^{!}} \alpha(z) \\
& A_{p, q}(z)=\frac{2(-z)^{p-1}}{(p)^{!}} A_{1, q}(z), \quad p \geq 2 \\
& A_{0, q}(z)=\frac{S^{2}(z)}{1-z} A_{1, q}(z)
\end{aligned}
$$

(ii) the generating functions $B_{p, q}(z)=\sum_{n=1}^{\infty} b_{p, q}^{n} z^{n}, p, q \geq 0$, are given by

$$
\begin{aligned}
& B_{1, q}(z)=\frac{(-z)^{q}}{(q)^{!}} G(z)-A_{1, q}(z) \\
& B_{p, q}(z)=\frac{2(-z)^{p-1}}{(p)^{!}} B_{1, q}(z)+\frac{(-z)^{p+q+2}}{(p)^{!}(q)^{!}} \sum_{k=2}^{p} \frac{2 k+1}{k(k+1)}, \quad p \geq 2, \\
& B_{0, q}(z)=\frac{S^{2}(z)}{1-z} B_{1, q}(z)+\frac{(-z)^{q}}{(q)^{!}} H(z)
\end{aligned}
$$

(iii) the generating functions $C_{p, q}(z)=\sum_{n=1}^{\infty} c_{p, q}^{n} z^{n}, p, q \geq 0$, are given by

$$
\begin{aligned}
& C_{0, q}(z)=d_{q} z^{q+2}+B_{0, q}(z)-\frac{(-z)^{q+2}}{(q)^{!}}, \\
& C_{1, q}(z)=\left[S^{1}(z)+\frac{z S^{2}(z)}{2(1-z)}\right] A_{1, q}(z)-\frac{z}{2} C_{0, q}(z), \\
& C_{p, q}(z)=\frac{2(-z)^{p-1}}{(p)^{!}} C_{1, q}(z), \quad p \geq 2,
\end{aligned}
$$


TABLE 1: Coefficients of the four-step transition kernel $K^{4}$, as given in (6).

\begin{tabular}{|c|c|c|c|c|c|c|c|c|c|c|c|c|}
\hline \multirow{3}{*}{$p$} & \multicolumn{4}{|c|}{ Values of $a_{p, q}^{4}$} & \multicolumn{3}{|c|}{ Values of $b_{p, q}^{4}$} & \multicolumn{5}{|c|}{ Values of $c_{p, q}^{4}$} \\
\hline & \multicolumn{12}{|c|}{$q$} \\
\hline & 0 & 1 & 2 & 3 & 0 & 1 & 2 & 3 & 0 & 1 & 2 & 3 \\
\hline 0 & $\frac{115}{96}$ & $-\frac{17}{36}$ & $\frac{1}{24}$ & 0 & $\frac{721}{432}$ & $-\frac{13}{12}$ & $\frac{1}{12}$ & 0 & $\frac{721}{432}$ & $-\frac{13}{12}$ & $\frac{5}{18}$ & 0 \\
\hline 1 & $\frac{11}{36}$ & $-\frac{1}{36}$ & $\frac{1}{12}$ & $-\frac{1}{144}$ & $-\frac{241}{540}$ & $\frac{17}{48}$ & $\frac{1}{36}$ & 0 & $-\frac{241}{540}$ & $\frac{17}{48}$ & $\frac{1}{36}$ & 0 \\
\hline 2 & $-\frac{11}{108}$ & $\frac{1}{12}$ & $-\frac{1}{72}$ & 0 & $\frac{3}{16}$ & $\frac{1}{36}$ & 0 & 0 & $-\frac{1}{144}$ & $\frac{1}{36}$ & 0 & 0 \\
\hline 3 & $\frac{1}{72}$ & $\frac{1}{144}$ & 0 & 0 & $\frac{1}{216}$ & 0 & 0 & 0 & $\frac{1}{216}$ & 0 & 0 & 0 \\
\hline 4 & $-\frac{1}{1440}$ & 0 & 0 & 0 & 0 & 0 & 0 & 0 & 0 & 0 & 0 & 0 \\
\hline
\end{tabular}

where the numbers $d_{q}$ are determined by their generating function $D(z)=\sum_{q=0}^{\infty} d_{q} z^{q}$ given by

$$
D(z)=\frac{1}{z}\left[\sqrt{z} J_{1}(2 \sqrt{z})(2 \gamma+\log z)-\pi \sqrt{z} Y_{1}(2 \sqrt{z})-1\right] .
$$

By the properties of generating functions, the coefficients $a_{p, q}^{n}, b_{p, q}^{n}$, and $c_{p, q}^{n}$ are determined from the derivatives of $A_{p, q}, B_{p, q}$, and $C_{p, q}$ evaluated at 0 . These derivatives are routinely calculated to any order with the help of computer algebra systems such as MATHEMATICA ${ }^{\circledR}$. Table 1 exemplifies the theorem by reporting the values of the coefficients $a_{p, q}^{n}, b_{p, q}^{n}$, and $c_{p, q}^{n}$ in the case $n=4$. Using these results, expression (5) for $\sigma^{2}$ can be evaluated explicitly in terms of certain integrals of hypergeometric functions; the computations, however, are quite involved and the final result rather lengthy, so we decided not to include them here.

\section{Proofs}

\subsection{Proofs of Proposition 1 and Theorem 1}

In this section we present the proofs of the relation between the Markov chains $M$ and $\Delta$, and of the central limit theorem.

Proof of Proposition 1. Since

$$
\begin{aligned}
\Delta_{n} & =l_{n}^{\prime}-l_{n} \\
& =\min \left\{l_{n-1}^{\prime}+Y_{n}, l_{n-1}+X_{n}+Z_{n}\right\}-\min \left\{l_{n-1}^{\prime}+Y_{n}+Z_{n}, l_{n-1}+X_{n}\right\} \\
& =\min \left\{\Delta_{n-1}+Y_{n-1}, X_{n-1}+Z_{n-1}\right\}-\min \left\{\Delta_{n-1}+Y_{n+1}+Z_{n-1}, X_{n-1}\right\},
\end{aligned}
$$

it follows at once that $M_{0}$ being equal to some $m^{\prime}=\left(r^{\prime}, x^{\prime}, y^{\prime}, z^{\prime}\right)$ implies that $\Delta_{1}=\min \left\{r^{\prime}+\right.$ $\left.y^{\prime}, x^{\prime}+z^{\prime}\right\}-\min \left\{r^{\prime}+y^{\prime}+z^{\prime}, x^{\prime}\right\}$ and, thus, the Markov property of $\Delta$ together with the independence of the edge weights implies that, for any integer $n>1$, the conditional probability $\mathrm{P}\left(M_{n} \in \mathrm{d} m \mid M_{0}=m^{\prime}\right)$ is given by

$$
\mathrm{e}^{-x+y+z} \mathrm{~d}^{3}(x, y, z) \mathrm{P}\left(\Delta_{n} \in \mathrm{d} r \mid \Delta_{1}=\min \left\{r^{\prime}+y^{\prime}, x^{\prime}+z^{\prime}\right\}-\min \left\{r^{\prime}+y^{\prime}+z^{\prime}, x^{\prime}\right\}\right) .
$$

The homogeneity of the Markov chain $\Delta$ then implies (6) because, for $n=1$, we clearly have

$$
\mathrm{P}\left(M_{1} \in \mathrm{d} m \mid M_{0}=m^{\prime}\right)=\mathrm{e}^{-(x+y+z)} \delta_{\min \left\{r^{\prime}+y^{\prime}, x^{\prime}+z^{\prime}\right\}-\min \left\{r^{\prime}+y^{\prime}+z^{\prime}, x^{\prime}\right\}}(r) \mathrm{d} m .
$$


Equation (3) about the stationary distribution of $M$ is a direct consequence of the fact that the edge weights $X_{n+1}, Y_{n+1}$, and $Z_{n+1}$ are independent of $\Delta_{n}$, and expression (4) was derived in [15, Proposition 5.5.].

Next, we prove the central limit theorem and the formula for the asymptotic variance $\sigma^{2}$.

Proof of Theorem 1. We apply the general result [7, Theorem 4.3] for functionals of ergodic Markov chains on general state spaces. We first note that

$$
\int_{\mathbb{R} \times \mathbb{R}_{+}^{3}} f(m)^{2} \tilde{\pi}(\mathrm{d} m)=\frac{2 J_{1}(2)-3 J_{0}(2)+{ }_{2} F_{3}(\{1,1\},\{2,2,2\} ;-1)-1}{J_{2}(2)}<\infty .
$$

It then suffices to prove that the Markov chain $M$ is uniformly ergodic, which is equivalent to showing that the Markov chain $\Delta$ is uniformly ergodic. We use the drift criterion [3, Theorem B], which asserts that if there is a sufficiently strong drift towards the center of the state space of a Markov chain, it is uniformly ergodic. Using the Lyapunov function $V(r)=1-\mathrm{e}^{-|r|}$ as well as

$$
K\left(r^{\prime}, r\right)= \begin{cases}\mathrm{e}^{-|r|} & \text { if } r^{\prime}<r<0 \vee r^{\prime}>r>0, \\ \mathrm{e}^{-\left|r^{\prime}-2 r\right|} & \text { otherwise }\end{cases}
$$

for the one-step transition kernel of $\Delta$ (see [15, Proposition 5.1]), we obtain

$$
\begin{aligned}
\psi(r) & :=P^{1} V(r) \\
& =1-\mathrm{E}_{r} \mathrm{e}^{-\left|\Delta_{1}\right|} \\
& =1-\int_{\mathbb{R}} \mathrm{e}^{-|\rho|} K(r, \rho) \mathrm{d} \rho \\
& =\frac{1}{6}\left[3-2 \mathrm{e}^{-|r|}+\mathrm{e}^{-2|r|}\right] .
\end{aligned}
$$

It is easy to check that

$$
\psi(r) \leq V(r)-\frac{1}{10}, \quad|r| \geq 1, \quad \text { and } \quad \sup _{|r| \leq 1} \psi(r) \leq \sup _{r \in \mathbb{R}} \psi(r)=\frac{1}{2}<\infty .
$$

Since the interval $[-1,1]$ is compact and has positive invariant measure, it is a small set (see [13, Remark 2.7]) and it follows that $\Delta$ is uniformly ergodic, which completes the proof.

\subsection{Summation formulae}

In this section we derive some summation formulae which we will use in the proofs in Subsection 3.3. Some of them are well known, others can be checked with computer algebra systems such as MATHEMATICA, a few (formulae 8, 9, 11, and 12) seem to be new. The sums will be evaluated explicitly in terms of Bessel and generalized hypergeometric functions, which we now define.

Definition 1. (Bessel function.) Let $\lambda$ be a real number in $\mathbb{R} \backslash \mathbb{Z}_{-}$. The Bessel function of the first kind of order $\lambda$, denoted by $J_{\lambda}$, is defined by the series representation

$$
J_{\lambda}(x)=\sum_{k=0}^{\infty} \frac{(-1)^{k}}{\Gamma(k) \Gamma(k+\lambda+1)}\left(\frac{x}{2}\right)^{2 k+\lambda} .
$$

For any integer $v$, the Bessel function of the second kind of order $v$, denoted by $Y_{v}$, is defined as

$$
Y_{\nu}(x)=\lim _{\lambda \rightarrow v} \frac{J_{\lambda}(x) \cos \lambda \pi-J_{-\lambda}(x)}{\sin \lambda \pi} .
$$


It is well known that Bessel functions satisfy the recurrence equation

$$
J_{v}(x)=\frac{2(v-1)}{x} J_{v-1}(x)-J_{v-2}(x)
$$

(see http://functions.wolfram.com/03.01.17.0002.01), which we use without further mentioning to simplify various expressions.

Definition 2. (Generalized hypergeometric function.) For nonnegative integers $p \leq q$ and complex numbers $\hat{a}=a_{1}, \ldots, a_{p}$ and $\hat{b}=b_{1}, \ldots, b_{q}, b_{j} \notin \mathbb{Z}_{-}$, the generalized hypergeometric function of order $(p, q)$ with coefficients $\hat{a}$ and $\hat{b}$, denoted by ${ }_{p} F_{q}(\hat{a}, \hat{b} ; \cdot)$, is defined by the series representation

$$
{ }_{p} F_{q}(\hat{a}, \hat{b} ; x)=\sum_{k=0}^{\infty} \frac{\left(a_{1}\right)_{k} \cdots\left(a_{p}\right)_{k}}{\left(b_{1}\right)_{k} \cdots\left(b_{q}\right)_{k}} \frac{x^{k}}{k !},
$$

where $(z)_{k}$ denotes the rising factorial defined by $(z)_{k}=\Gamma(z+k) / \Gamma(z)$.

In particular, we will encounter the regularized confluent hypergeometric functions ${ }_{0} \tilde{F}_{1}$, which are defined by ${ }_{0} \tilde{F}_{1}(\{\},\{b\} ;-z)={ }_{0} F_{1}(\{\},\{b\} ;-z) / \Gamma(b)$; in the next lemma we relate their derivative with respect to $b$ to certain sums involving the harmonic numbers $H_{k}:=$ $\sum_{n=1}^{k} 1 / n$.

Lemma 1. Denote by ${ }_{0} \tilde{F}_{1}$ the regularized version of the hypergeometric function ${ }_{0} F_{1}$. It then holds that

(i) for every positive integer $v$,

$$
\left.\frac{\mathrm{d}}{\mathrm{d} b}{ }_{0} \tilde{F}_{1}(\{\},\{b\} ;-z)\right|_{b=v}=\gamma z^{(1-v) / 2} J_{\nu-1}(2 \sqrt{z})-\sum_{k=0}^{\infty} \frac{(-z)^{k}}{k !(k+v-1) !} H_{k+v-1}
$$

(ii) for every positive integer $v$,

$$
\begin{aligned}
\left.\frac{\mathrm{d}}{\mathrm{d} b}{ }_{0} \tilde{F}_{1}(\{\},\{b\} ;-z)\right|_{b=-v}= & (-1)^{\nu-1} \gamma z^{(v+1) / 2} J_{v+1}(2 \sqrt{z})-\sum_{k=0}^{\infty} \frac{(-z)^{k+v+1}}{k !(k+v+1) !} H_{k} \\
& +(-1)^{v} \sum_{k=0}^{v} \frac{z^{k}(v-k) !}{k !} .
\end{aligned}
$$

Proof. For part (i), we differentiate the series representation (12) term by term. Using the definition of the digamma function $\digamma$ as the logarithmic derivative of the gamma function $\Gamma$ as well as the relation $\digamma(k)=-\gamma+H_{k-1}, k \in \mathbb{Z}_{+}$(see http://functions.wolfram.com/06.14.27. 0003.01), we obtain

$$
\left.\frac{\mathrm{d}}{\mathrm{d} b} \frac{1}{\Gamma(k+b)}\right|_{b=v}=-\frac{\digamma(k+v)}{\Gamma(k+v)}=\frac{\gamma-H_{k+v-1}}{(k+v-1) !}
$$

and, thus,

$$
\left.\frac{\mathrm{d}}{\mathrm{d} b}{ }_{0} \tilde{F}_{1}(\{\},\{b\} ;-z)\right|_{b=v}=\gamma \sum_{k=0}^{\infty} \frac{(-z)^{k}}{k !(k+v-1) !}-\sum_{k=0}^{\infty} \frac{(-z)^{k}}{k !(k+v-1) !} H_{k+v-1},
$$


which concludes the proof of the first part of the lemma. Part (ii) is shown in a completely analogous way, using the relation $\lim _{\mu \rightarrow-m} \digamma(\mu) / \Gamma(\mu)=(-1)^{m+1} m$ ! for every nonnegative integer $m$, which follows from the fact (see http://functions.wolfram.com/06.05.04.0004.01) that the gamma function has a simple pole at $-m$ with residue $(-1)^{m} / m$ !.

\section{Formula 1.}

$$
\sum_{k=1}^{\infty} \frac{(-z)^{k}}{k !(k+2) !}=\frac{2 J_{2}(2 \sqrt{z})-z}{2 z}=-\frac{1}{2} S^{1}(z) .
$$

Proof. The proof is immediate from the definition (11) of the Bessel function, given in (11).

\section{Formula 2.}

$$
\sum_{k=1}^{\infty} \frac{(-z)^{k}}{(k+1) !^{2}}=\frac{1-z-J_{0}(2 \sqrt{z})}{z}=-\frac{1}{2} S^{2}(z) .
$$

Proof. This is also clear from the definition of the Bessel function given in (11).

Formula 3.

$$
S^{3}(z)=\sum_{n=q+2}^{\infty} \frac{(-z)^{n-q}}{(n-q-2) !(n-q)^{2}}=1-J_{2}(2 \sqrt{z})-\sqrt{z} J_{1}(2 \sqrt{z}) .
$$

Proof. Shifting the index of summation $n$ by $q+2$ we obtain

$$
\begin{aligned}
S^{3}(z) & =\sum_{n=0}^{\infty} \frac{(-z)^{n+2}}{n !(n+1) !(n+2)^{2}} \\
& =\sum_{n=0}^{\infty}(-z)^{n+2}\left[\frac{1}{(n+1) !(n+2) !}-\frac{1}{(n+2) !^{2}}\right] \\
& =\sum_{n=0}^{\infty} \frac{(-z)^{n+1}}{(n)^{!}}+z-\sum_{n=0}^{\infty} \frac{(-z)^{n}}{n !^{2}}+1-z \\
& =1-J_{2}(2 \sqrt{z})-\sqrt{z} J_{1}(2 \sqrt{z}),
\end{aligned}
$$

by the definition of the Bessel function given in (11).

\section{Formula 4.}

$$
\Sigma^{1}(\zeta z)=\sum_{q=0}^{\infty} \sum_{k=0}^{q-2} \frac{(-\zeta z)^{q}}{(k)^{!}(q-k-2)^{!}(k+2)^{2}}=\frac{J_{1}(2 \sqrt{\zeta z})}{\sqrt{\zeta z}} S^{3}(\zeta z)
$$

Proof. By Fubini's theorem we can interchange the order of summation and then shift the summation index $q$ by $k+2$ to obtain

$$
\Sigma^{1}(\zeta z)=\sum_{k=0}^{\infty} \frac{(-\zeta z)^{k+2}}{(k)^{!}(k+2)^{2}} \sum_{q=0}^{\infty} \frac{(-\zeta z)^{q}}{(q)^{!}}
$$

By definition (11), the second sum equals $J_{1}(2 \sqrt{\zeta z}) / \sqrt{\zeta z}$ and so the claim follows with Formula 3. 


\section{Formula 5.}

$$
\Sigma^{2}(\zeta z)=\sum_{q=0}^{\infty} \sum_{k=1}^{q-2} \frac{2(-\zeta z)^{q}}{k !(k+2) !(q-k-2) !}=\left[2 J_{2}(2 \sqrt{\zeta z})-\zeta z\right] \sqrt{\zeta z} J_{1}(2 \sqrt{\zeta z})
$$

Proof. We can interchange the order of summation and shift the index $q$ by $k+2$ to obtain

$$
\Sigma^{2}(\zeta z)=\sum_{k=1}^{\infty} \frac{2(-\zeta z)^{k+2}}{k !(k+2) !} \sum_{q=0}^{\infty} \frac{(-\zeta z)^{q}}{(q)^{!}}
$$

The first factor equals $2 \zeta z J_{2}(2 \sqrt{\zeta z})-(\zeta z)^{2}$ and the second factor equals $J_{1}(2 \sqrt{\zeta z}) / \sqrt{\zeta z}$, both by definition (11), and so the claim follows.

\section{Formula 6.}

$$
T^{1}(\zeta z)=\sum_{q=0}^{\infty} \frac{(-\zeta z)^{q}}{(q)^{!}}=\frac{J_{1}(2 \sqrt{\zeta z})}{\sqrt{\zeta z}} .
$$

Proof. The proof is clear from definition (11).

\section{Formula 7.}

$$
T^{2}(\zeta z)=\sum_{q=0}^{\infty} \frac{(-\zeta z)^{q+1} q}{(q+1) !^{2}}=1-J_{0}(2 \sqrt{\zeta z})-\sqrt{\zeta z} J_{1}(2 \sqrt{\zeta z})
$$

Proof. This follows from the decomposition $q /(q+1) !^{2}=1 /(q)^{!}-1 /(q+1) !^{2}$ and definition (11).

\section{Formula 8 .}

$$
\begin{aligned}
U^{1}(z)= & \sum_{k=1}^{\infty} \frac{(-z)^{k+2}}{k !(k+2) !} \sum_{l=2}^{k} \frac{2 l+1}{l(l+1)} \\
= & \frac{3 z^{2}}{4}-z-2-\pi z Y_{2}(2 \sqrt{z})+\frac{\sqrt{z}}{2} J_{1}(2 \sqrt{z})[4 \gamma-3+2 \log z] \\
& +J_{0}(2 \sqrt{z})\left[1+\frac{5 z}{2}-2 \gamma z-z \log z\right]
\end{aligned}
$$

Proof. First we note that

$$
\sum_{l=2}^{k} \frac{2 l+1}{l(l+1)}=\sum_{l=2}^{k}\left[\frac{1}{l}+\frac{1}{l+1}\right]=2 H_{k}-\frac{5 k+3}{2(k+1)}
$$

where $H_{k}$ denotes the $k$ th harmonic number. The first contribution to $U^{1}(z)$ can therefore be computed as

$$
\begin{aligned}
\frac{1}{2} \sum_{k=1}^{\infty} \frac{(5 k+3)(-z)^{k}}{k !(k+2) !(k+1)} & =-\frac{1}{z}-\frac{3}{4}-\frac{1}{z} \sum_{k=0}^{\infty} \frac{(-z)^{k}}{(k)^{!}}+\frac{5}{2} \sum_{k=0}^{\infty} \frac{(-z)^{k}}{k !(k+2) !} \\
& =\frac{1}{4 z^{3 / 2}}\left[4 J_{1}(2 \sqrt{z})+10 \sqrt{z} J_{2}(2 \sqrt{z})-3 z^{3 / 2}-4 \sqrt{z}\right]
\end{aligned}
$$


The other contribution to $U^{1}(z)$ is, with the help of Lemma 1(ii), obtained as

$$
2 \sum_{k=1}^{\infty} \frac{(-z)^{k}}{k !(k+2) !} H_{k}=\frac{2}{z^{2}}\left[\gamma z J_{2}(2 \sqrt{z})-z-1-\left.\frac{\mathrm{d}}{\mathrm{d} b}{ }_{0} \tilde{F}_{1}(\{\},\{b\} ;-z)\right|_{b=-1}\right] .
$$

By known properties of the regularized confluent hypergeometric function (see http://functions. wolfram.com/07.18.20.0015.01),

$$
\left.\frac{\mathrm{d}}{\mathrm{d} b}{ }_{0} \tilde{F}_{1}(\{\},\{b\} ;-z)\right|_{b=-1}=\frac{1}{2}\left[\pi z Y_{2}(2 \sqrt{z})-\sqrt{z} J_{1}(2 \sqrt{z})[2+\log z]+J_{0}(2 \sqrt{z})[z \log z-1]\right],
$$

and the result follows upon combining the last four displayed equations.

\section{Formula 9.}

$$
\Upsilon^{1}(\zeta z)=\sum_{q=0}^{\infty} \sum_{k=1}^{q-2} \frac{(-\zeta z)^{q}}{(q-k-2)^{!} k !(k+2) !} \sum_{l=2}^{k} \frac{2 l+1}{l(l+1)}=\frac{J_{1}(2 \sqrt{\zeta z})}{\sqrt{\zeta z}} U^{1}(\zeta z) .
$$

Proof. Interchanging the order of the first two summations and shifting the index $q$ by $k+2$ we find that

$$
\Upsilon^{1}(\zeta z)=\sum_{k=1}^{\infty} \frac{(-\zeta z)^{k+2}}{k !(k+2) !} \sum_{q=0}^{\infty} \frac{(-\zeta z)^{q}}{(q)^{!}} \sum_{l=2}^{k} \frac{2 l+1}{l(l+1)}
$$

As before, the sum in the middle equals $J_{1}(2 \sqrt{\zeta z}) / \sqrt{\zeta z}$ and so the claim follows with Formula 8 .

\section{Formula 10.}

$$
S^{4}(z)=\sum_{n=q+2}^{\infty} \frac{(-z)^{n-q}}{(n-q-2)^{!}(n-q-1)^{2}}=z_{2}^{2} F_{3}(\{1,1\},\{2,2,2\} ;-z) .
$$

Proof. After shifting the index $n$ by $q+2$, the proof follows immediately from the definition of the hypergeometric function, given in (12).

\section{Formula 11.}

$$
\begin{aligned}
U^{2}(z)= & \sum_{k=1}^{\infty} \frac{(-z)^{k+2}}{(k+1) !^{2}} \sum_{l=2}^{k} \frac{2 l+1}{l(l+1)} \\
=\frac{z}{2} & {\left[-5+3 z+2 \pi Y_{0}(2 \sqrt{z})-2 z_{2} F_{3}(\{1,1\},\{2,2,2\},-z)\right.} \\
& \left.\quad+J_{0}(2 \sqrt{z})(5-4 \gamma-2 \log z)\right] .
\end{aligned}
$$

Proof. The proof is analogous to that of Formula 8. Using (13), the first contribution to $U^{2}(z)$ is

$$
\begin{aligned}
\frac{1}{2} \sum_{k=1}^{\infty} \frac{(5 k+3)(-z)^{k+2}}{(k+1) !^{2}(k+1)} & =\frac{5-3 z}{2 z}-\frac{5}{2 z} \sum_{k=0}^{\infty} \frac{(-z)^{k}}{k !^{2}}-\sum_{k=0}^{\infty} \frac{(-z)^{k}}{(k+1) !^{2}(k+1)^{2}} \\
& =\frac{1}{2 z}\left[5-3 z-5 J_{0}(2 \sqrt{z})-2 z_{2} F_{3}(\{1,1\},\{2,2,2\} ;-z)\right],
\end{aligned}
$$


where we used definitions (11) and (12). For the remaining part, we first use Lemma 1(i) and the identity $J_{1}(2 \sqrt{z}) / \sqrt{z}-J_{2}(2 \sqrt{z})=J_{0}(2 \sqrt{z})$ to compute

$$
\begin{aligned}
& \sum_{k=1}^{\infty} \frac{(-z)^{k}}{(k+1) !^{2}} H_{k+2} \\
& \quad=\sum_{k=1}^{\infty} \frac{(-z)^{k}}{(k+1) !(k+2) !} H_{k+2}+\sum_{k=1}^{\infty} \frac{(-z)^{k}}{k !(k+2) !} H_{k+2} \\
& \quad=-\frac{3}{2}+\frac{1}{z}-\frac{1}{z}\left[\sum_{k=0}^{\infty} \frac{(-z)^{k}}{(k)^{!}} H_{k+1}-z \sum_{k=0}^{\infty} \frac{(-z)^{k}}{k !(k+2) !} H_{k+2}\right] \\
& \quad=-\frac{3}{2}+\frac{1}{z}-\frac{\gamma}{z} J_{0}(2 \sqrt{z})+\frac{1}{z}\left[\left.\frac{\mathrm{d}}{\mathrm{d} b} 0 \tilde{F}_{1}(\{\},\{b\} ;-z)\right|_{b=2}-\left.z \frac{\mathrm{d}}{\mathrm{d} b}{ }_{0} \tilde{F}_{1}(\{\},\{b\} ;-z)\right|_{b=3}\right]
\end{aligned}
$$

It then follows from the explicit characterization of $\left.(\mathrm{d} / \mathrm{d} b)_{0} \tilde{F}_{1}(\{\},\{b\} ;-z)\right|_{b=-v}, v \in \mathbb{Z}_{+}$(see http://functions.wolfram.com/07.18.20.0013.01), that

$$
\sum_{k=1}^{\infty} \frac{(-z)^{k}}{(k+1) !^{2}} H_{k+2}=\frac{1}{2 z^{3 / 2}}\left[\sqrt{z}\left[2-3 z+\pi Y_{0}(2 \sqrt{z})\right]-2 J_{1}(2 \sqrt{z})-\sqrt{z} J_{0}(2 \sqrt{z})[2 \gamma+\log z]\right]
$$

Using this, we obtain

$$
\begin{aligned}
2 \sum_{k=1}^{\infty} & \frac{(-z)^{k}}{(k+1) !^{2}} H_{k} \\
= & 2 \sum_{k=1}^{\infty} \frac{(-z)^{k}}{(k+1) !^{2}} H_{k+2}-2 \sum_{k=1}^{\infty} \frac{(-z)^{k}}{(k+1) !^{2}(k+2)}-2 \sum_{k=1}^{\infty} \frac{(-z)^{k}}{(k+1) !^{2}(k+1)} \\
= & \frac{1}{2 z^{3 / 2}}\left[\sqrt{z}\left[2-3 z+\pi Y_{0}(2 \sqrt{z})\right]-2 J_{1}(2 \sqrt{z})-\sqrt{z} J_{0}(2 \sqrt{z})[2 \gamma+\log z]\right] \\
& +\left[\frac{1}{2}-\frac{1}{z}+\frac{J_{1}(2 \sqrt{z})}{z^{3 / 2}}\right]+1-{ }_{2} F_{3}(\{1,1\},\{2,2,2\} ;-z) .
\end{aligned}
$$

Combining (14) and (15) completes the proof.

\section{Formula 12.}

$$
\Upsilon^{2}(\zeta z)=\sum_{q=0}^{\infty} \sum_{k=1}^{q-1} \frac{(-\zeta z)^{q}}{(q-k-1)^{!}(k+1) !^{2}} \sum_{l=2}^{k} \frac{2 l+1}{l(l+1)}=-\frac{J_{1}(2 \sqrt{\zeta z})}{(\zeta z)^{3 / 2}} U^{2}(\zeta z) .
$$

Proof. Interchanging the order of the first two summations and shifting the index $q$ by $k+1$ we find that

$$
\Upsilon^{2}(\zeta z)=\sum_{k=1}^{\infty} \frac{(-\zeta z)^{k+2}}{(k+1) !^{2}} \sum_{q=0}^{\infty} \frac{(-\zeta z)^{q-1}}{(q)^{!}} \sum_{l=2}^{k} \frac{2 l+1}{l(l+1)} .
$$

By definition (11), the middle sum is equal to $-J_{1}(2 \sqrt{\zeta z}) /(\zeta z)^{3 / 2}$ and so the result follows readily from Formula 11 . 


\section{Formula 13.}

$$
\Sigma^{3}(\zeta z)=\sum_{q=0}^{\infty} \sum_{k=1}^{q-1} \frac{2(-\zeta z)^{q}}{(q-k-1) !(k+1) !^{2}}=\frac{2\left[\zeta z-1+J_{0}(2 \sqrt{\zeta z})\right] J_{1}(2 \sqrt{\zeta z})}{\sqrt{\zeta z}} .
$$

Proof. The proof is the same as that of Formula 5 and so we omit it.

\section{Formula 14.}

$$
\Sigma^{4}(\zeta z)=\sum_{q=0}^{\infty} \sum_{k=0}^{q-1} \frac{(-\zeta z)^{q}}{(k)^{!}(q-k-1)^{!}(k+1)^{2}}=-\sqrt{\zeta z} J_{1}(2 \sqrt{\zeta z})_{2} F_{3}(\{1,1\},\{2,2,2\} ;-\zeta z)
$$

Proof. Using Fubini's theorem, we obtain

$$
\Sigma^{4}(\zeta z)=\sum_{k=0}^{\infty} \frac{(-\zeta z)_{k}}{(k)^{!}(k+1)^{2}} \sum_{q=k+1}^{\infty} \frac{(-\zeta z)^{q-k}}{(q-k-1) !}
$$

The first factor is equal to ${ }_{2} F_{3}(\{1,1\},\{2,2,2\} ;-\zeta z)$ by definition (12) and the second factor equals $-\sqrt{\zeta z} J_{1}(2 \sqrt{\zeta z})$ by definition (11).

Having evaluated these sums we now turn to relations between them which we will also need, and which are proved by writing out the expressions and straightforward computations. The first relation involves only the five functions appearing in the statement of Theorem 2.

Lemma 2. For almost every complex number $z$ with respect to the Lebesgue measure on the complex plane,

$$
\left[\frac{S^{2}(z)}{2(1-z)}+\frac{1}{z}\right] G(z)+\left[\frac{S^{1}(z)}{z}+\frac{S^{2}(z)}{1-z}+\frac{1}{z}\right] \alpha(z)+\frac{H(z)}{2}+\frac{3 z^{2}}{4}=0 .
$$

Lemma 3. For almost every complex number $z$ with respect to the Lebesgue measure on the complex plane,

$$
U^{1}(z)+S^{3}(z)+\left[\frac{S^{1}(z)-1}{z}\right] G(z)=\frac{3}{4} z^{2}-z-1 .
$$

\subsection{Proof of Theorem 2}

In this section we prove the main result, Theorem 2. First, however, we take a closer look at the coefficients $a_{p, q}^{n}, b_{p, q}^{n}$, and $c_{p, q}^{n}$ which are defined implicitly through the generating functions (7), (8), and (9).

Lemma 4. For any integers $p, q \geq 0$, the following relations hold:

$$
a_{p, q}^{1}=\delta_{p, 1} \delta_{q, 0}, \quad b_{p, q}^{1}=0, \quad c_{p, q}^{1}=0 .
$$

Proof. The proof follows from evaluating the derivatives of the generating functions $A_{p, q}$, $B_{p, q}$, and $C_{p, q}$ at 0 .

Next we derive some useful relations between the coefficients $a_{p, q}^{n}, b_{p, q}^{n}$, and $c_{p, q}^{n}$. These will be the main ingredient in our inductive proof of Theorem 2. The general strategy in proving the equality of two sequences $\left(s_{n}\right)_{n \geq 1}$ and $\left(\tilde{s}_{n}\right)_{n \geq 1}$ will be to compute their generating functions $\sum_{n \geq 1} s_{n} z^{n}$ and $\sum_{n \geq 1} \tilde{s}_{n} z^{n}$, and to show that they coincide for every $z$. The validity 
of this approach follows from the well-known bijection between sequences of real numbers and generating functions (see, e.g. [20] for an introductory treatment). We will constantly be making use of the convolution property of generating functions. By this we mean the simple fact that if $\left(s_{n}\right)_{n \geq 1}$ is a real sequence with generating function $\bar{S}(z)$, and $\left(t_{n}\right)_{n \geq 1}$ is another such sequence with generating function $\bar{T}(z)$, then the sequence of partial sums $\left(\sum_{\nu=1}^{n-1} t_{n-v} s_{v}\right)_{n \geq 1}$ has generating function $\bar{S}(z) \bar{T}(z)$. We also encounter generating functions of sequences indexed by $q$ instead of $n$. In this case we denote the formal variable by $\zeta$ instead of $z$ and sums are understood to be indexed from 0 to $\infty$.

Lemma 5. For all integers $n \geq 1$ and $q \geq 0$, the coefficients defined by the generating functions given in Theorem 2 satisfy the relation

$$
\sum_{k=0}^{q-2} \frac{b_{k, q-k-2}^{n}}{k+2}-\sum_{k=0}^{q-2} \frac{c_{k, q-k-2}^{n}}{k+2}=\delta_{q, n}\left[\frac{(-1)^{n}(n-1)}{n !^{2}}-\sum_{k=0}^{n-2} \frac{(-1)^{n}}{(k)^{!}(n-k-2)^{!}(k+2)^{2}}\right] .
$$

Proof. These equations are true for all $n \geq 1$ if and only if the corresponding generating functions coincide. Multiplying both sides by $z^{n}$, summing over $n$, and using the recursive definitions of the generating functions as well as the facts that, by Formulae 1 and 2 ,

$$
\sum_{k=1}^{\infty} \frac{(-z)^{k}}{k !(k+2) !}=-\frac{1}{2} S^{1}(z), \quad \sum_{k=1}^{\infty} \frac{(-z)^{k}}{(k+1) !^{2}}=-\frac{1}{2} S^{2}(z)
$$

we find that the claim of the lemma is equivalent to

$$
\begin{aligned}
0= & \sum_{k=1}^{q-2} \frac{(-z)^{q}}{k !(k+2) !(q-k-2)^{!}} \sum_{l=1}^{k} \frac{2 l+1}{l(l+1)}+\frac{1}{2} \Theta_{q, 2}\left[\frac{(-z)^{q}}{(q-2)^{!}}-z^{q} d_{q-2}\right] \\
& +\frac{z^{q}}{2} \sum_{k=1}^{q-2} \frac{2(-1)^{k-1}}{k !(k+2) !} d_{q-k-2}-\Theta_{q, 1}\left[\frac{(-z)^{q}(q-1)}{q !^{2}}-\sum_{k=0}^{q-2} \frac{(-z)^{q}}{(k)^{!}(q-k-2)^{!}(k+2)^{2}}\right] \\
& +\frac{5}{4} z^{3} \sum_{k=1}^{q-2} \frac{2(-z)^{q-3}}{k !(k+2) !(q-k-2) !},
\end{aligned}
$$

where we have used Lemma 2 to simplify the coefficient of the last sum. To show this equality for all nonnegative integers $q$, we compare the $q$-generating functions and must then show that

$$
0=\Upsilon^{1}(\zeta z)+\frac{(\zeta z)^{2}}{2}\left[T^{1}(\zeta z)+\left(S^{1}(\zeta z)-1\right) D(\zeta z)\right]-T^{2}(\zeta z)+\Sigma^{1}(\zeta z)+\frac{5}{4} \Sigma^{2}(\zeta z)
$$

where closed-form expressions for

$$
\begin{aligned}
\Sigma^{1}(\zeta z) & :=\sum_{q=0}^{\infty} \sum_{k=0}^{q-2} \frac{(-\zeta z)^{q}}{(k)^{!}(q-k-2)^{!}(k+2)^{2}} \\
\Sigma^{2}(\zeta z) & :=\sum_{q=0}^{\infty} \sum_{k=1}^{q-2} \frac{2(-\zeta z)^{q}}{k !(k+2) !(q-k-2)^{!}}, \\
T^{1}(\zeta z) & :=\sum_{q=0}^{\infty} \frac{(-\zeta z)^{q}}{(q)^{!}}
\end{aligned}
$$




$$
T^{2}(\zeta z):=\sum_{q=0}^{\infty} \frac{(-\zeta z)^{q+1} q}{(q+1) !^{2}}
$$

and

$$
\Upsilon^{1}(\zeta z):=\sum_{q=0}^{\infty} \sum_{k=1}^{q-2} \frac{(-\zeta z)^{q}}{(q-k-2) ! k !(k+2) !} \sum_{l=2}^{k} \frac{2 l+1}{l(l+1)}
$$

are derived in Formulae 4, 5, 6, 7, and 9. Using these closed-form formulae, (16) is seen to be identically true by simple algebra.

Lemma 6. For all integers $p, q \geq 0$, the sequences of coefficients defined by the generating functions given in Theorem 2 satisfy the recursion

$$
\begin{aligned}
& a_{p, q}^{n+1}= \delta_{p, 0} \sum_{k=0}^{n} \frac{a_{k, q}^{n}}{k+1}-\Theta_{p, 1} \frac{a_{p-1, q}^{n}}{p(p+1)} \\
&+\delta_{p, 1}\left[\tilde{\Theta}_{q, n-2} \frac{(-1)^{n}}{(n-q-2)^{!}(q)^{!}(n-q)^{2}}+\delta_{q, n-1} \frac{(-1)^{n-1}}{(n-1)^{!}}\right. \\
&-\delta_{q, n}\left[\sum_{k=0}^{n-2} \frac{(-1)^{n}}{(k)^{!}(n-k-2)^{!}(k+2)^{2}}+\frac{(-1)^{n-1} n}{(n-1) !(n+1) !}\right] \\
&\left.+\sum_{k=0}^{n} \frac{b_{k, q}^{n}}{k+2}-\sum_{k=0}^{q-2} \frac{b_{k, q-k-2}^{n}}{k+2}+\sum_{k=0}^{q-2} \frac{c_{k, q-k-2}^{n}}{k+2}\right], \quad n \geq 1 .
\end{aligned}
$$

Proof. Applying Lemma 5 and computing the generating functions of both sides of the asserted equality, we find that the claim of the lemma is equivalent to

$$
\begin{aligned}
& \frac{1}{z} A_{p, q}(z)=\delta_{p, 0} \frac{S^{2}(z)}{z(1-z)} A_{1, q}(z)-\Theta_{p, 1} \frac{A_{p-1, q}(z)}{p(p+1)} \\
&+\delta_{p, 1} \frac{(-z)^{q}}{(q)^{!}}[\left(\frac{S^{2}(z)}{2(1-z)}+\frac{S^{1}(z)}{z}\right)(G(z)-\alpha(z)) \\
&\left.+\frac{H(z)}{2}+S^{3}(z)+U^{1}(z)+z+1\right],
\end{aligned}
$$

where explicit expressions for

$$
S^{3}(z):=\sum_{n=q+2}^{\infty} \frac{(-z)^{n-q}}{(n-q-2)^{!}(n-q)^{2}}
$$

and

$$
U^{1}(z):=\sum_{k=1}^{\infty} \frac{(-z)^{k+2}}{k !(k+2) !} \sum_{l=2}^{k} \frac{2 l+1}{l(l+1)}
$$

are derived in Formula 3 and Formula 8. For $p=0$ and $p>1$, (17) follows immediately from the defining equations (7c) and (7b). For $p=1$, the claim follows from combining Lemmas 2 and 3 . 
Lemma 7. For all integers $p, q \geq 0$, the sequences of coefficients defined by the generating functions given in Theorem 2 satisfy the recursion

$$
\begin{aligned}
b_{p, q}^{n+1}= & \delta_{p, 1} \sum_{k=0}^{n} \frac{a_{k, q}^{n}}{k+2} \\
& +\delta_{p, 0}\left[\delta_{q, n-1} \frac{(-1)^{n-1}}{(n-1)^{!}}+\tilde{\Theta}_{q \cdot n-2} \frac{(-1)^{n}}{(n-q-2)^{!}(q)^{!}(n-q-1)^{2}}+\sum_{k=0}^{n} \frac{b_{k, q}^{n}}{k+1}\right] \\
& -\Theta_{p, 1}\left[\frac{b_{p-1, q}^{n}}{p(p+1)}+\delta_{q, n-p-1} \frac{(-1)^{n}(2 p+1)}{(p)^{!}(n-p-1)^{!} p(p+1)}\right], \quad n \geq 1 .
\end{aligned}
$$

Proof. We proceed as in the proof of Lemma 6 and show the equality for every $n$ by showing the equality of the generating functions. We find that the claim is equivalent to

$$
\begin{aligned}
\frac{B_{p, q}(z)}{z}= & \delta_{p, 1}\left[\frac{S^{1}(z)}{z}+\frac{S^{2}(z)}{2(1-z)}\right] A_{1, q}(z) \\
& +\delta_{p, 0} \frac{(-z)^{q+1}}{(q)^{!}}\left[S^{4}(z)+U^{2}(z)+z+\frac{S^{2}(z)}{z(1-z)}[G(z)-\alpha(z)]+H(z)\right] \\
& -\Theta_{p .1}\left[\frac{B_{p-1, q}(z)}{p(p+1)}+\frac{(-z)^{p+q+1}(2 p+1)}{(p)^{!}(q)^{!} p(p+1)}\right],
\end{aligned}
$$

where the functions

$$
S^{4}(z):=\sum_{n=q+2}^{\infty} \frac{(-z)^{n-q}}{(n-q-2)^{!}(n-q-1)^{2}} \quad \text { and } \quad U^{2}(z):=\sum_{k=1}^{\infty} \frac{(-z)^{k+2}}{(k+1) !^{2}} \sum_{l=2}^{k} \frac{2 l+1}{l(l+1)}
$$

are evaluated in Formulae 10 and 11. For $p=0,(18)$ follows from the observation that

$$
H(z)=z\left[S^{4}(z)+U^{2}(z)+z+H(z)\right] .
$$

Next we observe that (8b) implies that

$$
\begin{aligned}
B_{p, q}(z)+\frac{z B_{p-1, q}(z)}{p(p+1)} & =\frac{(-z)^{p+q+2}}{(p)^{!}(q)^{!}} \sum_{k=2}^{p} \frac{2 k+1}{k(k+1)}-\frac{(-z)^{p+q+2}}{(p)^{!}(q)^{!}} \sum_{k=2}^{p-1} \frac{2 k+1}{k(k+1)} \\
& =\frac{(-z)^{p+q+2}}{(p)^{!}(q)^{!}} \frac{2 p+1}{p(p+1)},
\end{aligned}
$$

and, thus, (18) also holds for $p>1$. Finally, for $p=1$, we need to show that

$$
\frac{B_{1, q}(z)}{z}=\left[\frac{S^{1}(z)}{z}+\frac{S^{2}(z)}{2(1-z)}\right] A_{1, q}(z)-\frac{B_{0, q}(z)}{2}-\frac{3(-z)^{q+1}}{4(q)^{!}},
$$

which, after using the defining equations (7) and (8) several times, amounts to showing that

$$
\left[\frac{S^{2}(z)}{2(1-z)}+\frac{1}{z}\right] G(z)+\left[\frac{S^{1}(z)}{z}+\frac{S^{2}(z)}{1-z}+\frac{1}{z}\right] \alpha(z)+\frac{H(z)}{2}+\frac{3 z^{2}}{4}=0,
$$

which is exactly what Lemma 2 asserts. 
Lemma 8. For all integers $p, q \geq 0$, the sequences of coefficients defined by the generating functions given in Theorem 2 satisfy the recursion

$$
\begin{aligned}
c_{p, q}^{n+1}= & \delta_{p, 1} \sum_{k=0}^{n} \frac{a_{k, q}^{n}}{k+2}-\Theta_{p, 1} \frac{c_{p-1, q}^{n}}{p(p+1)} \\
+\delta_{p, 0}\left[\tilde{\Theta}_{q, n-2} \frac{(-1)^{n}}{(n-q-2)^{!}(q)^{!}(n-q-1)^{2}}\right. & \\
& +\delta_{q, n-1}\left(\sum_{k=0}^{n-2} \frac{(-1)^{n}}{(k)^{!}(n-k-2)^{!}(k+1)^{2}}-\frac{(-1)^{n-1}}{n !^{2}}\right) \\
& \left.+\sum_{k=0}^{n} \frac{b_{k, q}^{n}}{k+1}-\sum_{k=0}^{q-1} \frac{b_{k, q-k-1}^{n}}{k+1}+\sum_{k=0}^{q-1} \frac{\left.c_{k, q-k-1}^{n}\right], \quad n \geq 1 .}{k+1}\right] \quad n \geq 1 .
\end{aligned}
$$

Proof. The proof follows along the same lines as the previous proofs. Equating the generating functions of both sides and using Lemma 2, we need to show that

$$
\begin{aligned}
& \frac{C_{p, q}(z)}{z}=\delta_{p, 1}\left[\frac{S^{1}(z)}{z}+\frac{S^{2}(z)}{2(1-z)}\right] A_{1, q}(z)-\Theta_{p, 1} \frac{C_{p-1, q}(z)}{p(p+1)} \\
&+\delta_{p, 0}\left[\frac{(-z)^{q}}{(q)^{!}}\left(\frac{S^{2}(z)}{z(1-z)}(G(z)-\alpha(z))+H(z)+S^{4}(z)+U^{2}(z)+\frac{z}{q+1}\right)\right. \\
&+\frac{5 z}{4} \sum_{k=1}^{q-1} \frac{2(-z)^{q}}{(q-k-1)^{!}(k+1) !^{2}}-\sum_{k=0}^{q-1} \frac{(-z)^{q+1}}{(k)^{!}(q-k-1)^{!}(k+1)^{2}} \\
&-\sum_{k=1}^{q-1} \frac{(-z)^{q+1}}{(q-k-1)^{!}(k+1) !^{2}} \sum_{l=2}^{k} \frac{2 l+1}{l(l+1)}-\frac{z^{q+1}}{2} \sum_{k=1}^{q-1} \frac{2(-1)^{k-1}}{(k+1) !^{2}} d_{q-k-1} \\
&\left.+\Theta_{q, 1}\left(z^{q+1} d_{q-1}-\frac{(-z)^{q+1}}{(q-1)^{!}}\right)\right]
\end{aligned}
$$

For $p \geq 1,(20)$ is immediately clear from the defining equations (9b) and (9c). For $p=0$, we show that the $q$-generating functions coincide. Doing this we find, after some algebra, that (19) is equivalent to

$$
\begin{aligned}
0= & {\left[\frac{z-1}{z} H(z)+S^{4}(z)+z+U^{2}(z)-\zeta z^{2}\right] T^{1}(\zeta z)+z\left[1-\frac{S^{2}(z)}{2}\right] } \\
& +\left[\zeta z^{2}\left(1-\frac{S^{2}(\zeta z)}{2}\right)-z\right] D(\zeta z)+\frac{5 z}{4} \Sigma^{3}(\zeta z)+z\left[\Sigma^{4}(\zeta z)+\Upsilon^{2}(\zeta z)\right]
\end{aligned}
$$

with the functions

$$
\begin{aligned}
& \Sigma^{3}(\zeta z)=\sum_{q=0}^{\infty} \sum_{k=1}^{q-1} \frac{2(-\zeta z)^{q}}{(q-k-1)^{!}(k+1) !^{2}}, \\
& \Sigma^{4}(\zeta z)=\sum_{q=0}^{\infty} \sum_{k=0}^{q-1} \frac{(-\zeta z)^{q}}{(k)^{!}(q-k-1)^{!}(k+1)^{2}},
\end{aligned}
$$


and

$$
\Upsilon^{2}(\zeta z)=\sum_{q=0}^{\infty} \sum_{k=1}^{q-1} \frac{(-\zeta z)^{q}}{(q-k-1)^{!}(k+1) !^{2}} \sum_{l=2}^{k} \frac{2 l+1}{l(l+1)}
$$

given in Formulae 13, 14, and 12. Since all functions occurring in (21) are explicitly known, the result follows from basic algebra.

We can now prove our main theorem.

Proof of Theorem 2. The Chapman-Kolmogorov equation implies the recursion

$$
K^{n}\left(r^{\prime}, r\right)=\int_{\mathbb{R}} K\left(r^{\prime}, s\right) K^{n-1}(s, r) \mathrm{d} s, \quad r, r^{\prime} \in \mathbb{R}, n>1,
$$

where $K$ is the one-step transition kernel of $\Delta$ given in (10). From this we can first prove the asserted symmetry $K^{n}\left(r^{\prime}, r\right)=K^{n}\left(-r^{\prime},-r\right)$ by induction on $n$. For $n=1$, this is clearly true, so assuming that it holds for some $n \geq 0$ we conclude that $K^{n+1}\left(r^{\prime}, r\right)$ is equal to

$$
\begin{aligned}
\int_{\mathbb{R}} K\left(r^{\prime}, s\right) K^{n}(s, r) \mathrm{d} s & =\int_{\mathbb{R}} K\left(-r^{\prime},-s\right) K^{n}(-s,-r) \mathrm{d} s \\
& =\int_{\mathbb{R}} K\left(-r^{\prime}, s\right) K^{n}(s,-r) \mathrm{d} s \\
& =K^{n+1}\left(-r^{\prime},-r\right) .
\end{aligned}
$$

In the next step we prove (6), also by induction on $n$. For $n=1$, the claim is true by Lemma 4 . We now assume that (6) holds for some $n \geq 1$. It then follows that, for $r \geq 0$,

$$
\begin{aligned}
K^{n+1}\left(r^{\prime}, r\right)= & \int_{\mathbb{R}} K\left(r^{\prime}, s\right) K^{n}(s, r) \mathrm{d} s \\
= & \int_{-\infty}^{0} K\left(r^{\prime}, s\right) K^{n}(s, r) \mathrm{d} s+\int_{0}^{r} K\left(r^{\prime}, s\right) K^{n}(s, r) \mathrm{d} s+\int_{r}^{\infty} K\left(r^{\prime}, s\right) K^{n}(s, r) \mathrm{d} s \\
= & \sum_{p, q=0}^{n} a_{p, q}^{n} \mathrm{e}^{-(q+2) r} \int_{-\infty}^{0} K\left(r^{\prime}, s\right) \mathrm{e}^{p s} \mathrm{~d} s+\frac{(-1)^{n-1} \mathrm{e}^{-(n+1) r}}{(n-1) !} \int_{0}^{r} K\left(r^{\prime}, s\right) \mathrm{e}^{s} \mathrm{~d} s \\
& +\sum_{p=0}^{n-1} \frac{(-1)^{n} \mathrm{e}^{-(n-p) r}}{(p)^{!}(n-p-2)^{!}} \int_{0}^{r} K\left(r^{\prime}, s\right) s \mathrm{e}^{-p s} \mathrm{~d} s \\
& +\sum_{p, q=0}^{n} b_{p, q}^{n} \mathrm{e}^{-(q+2) r} \int_{0}^{r} K\left(r^{\prime}, s\right) \mathrm{e}^{-p s} \mathrm{~d} s \\
& +\frac{(-1)^{n-1} \mathrm{e}^{-r}}{(n-1)^{!}} \int_{r}^{\infty} K\left(r^{\prime}, s\right) \mathrm{e}^{-(n-1) s} \mathrm{~d} s \\
& +\sum_{p=0}^{n-1} \frac{(-1)^{n} \mathrm{e}^{-(n-p) r} r}{(p)^{!}(n-p-2)^{!}} \int_{r}^{\infty} K\left(r^{\prime}, s\right) \mathrm{e}^{-p s} \mathrm{~d} s \\
& +\sum_{p, q=0}^{n} c_{p, q}^{n} \mathrm{e}^{-(q+2) r} \int_{r}^{\infty} K\left(r^{\prime}, s\right) \mathrm{e}^{-p s} \mathrm{~d} s .
\end{aligned}
$$


The five types of integral occurring in this expression are easily evaluated to give, for $p \geq 0$,

$$
\begin{aligned}
& \int_{-\infty}^{0} K\left(r^{\prime}, 2\right) \mathrm{e}^{p s} \mathrm{~d} s= \begin{cases}\frac{1}{p+1}-\frac{\mathrm{e}^{(p+1) r^{\prime}}}{(p+1)(p+2)}, & r^{\prime} \leq 0, \\
\frac{\mathrm{e}^{-r^{\prime}}}{p+2}, & r^{\prime}>0,\end{cases} \\
& \int_{0}^{r} K\left(r^{\prime}, 2\right) \mathrm{e}^{s} \mathrm{~d} s= \begin{cases}\mathrm{e}^{r^{\prime}}-\mathrm{e}^{r^{\prime}-r}, & r^{\prime} \leq 0, \\
1-\mathrm{e}^{r^{\prime}-r}+r^{\prime}, & 0<r^{\prime} \leq r, \\
r, & r^{\prime}>r,\end{cases} \\
& \int_{0}^{r} K\left(r^{\prime}, 2\right) s \mathrm{e}^{-p s} \mathrm{~d} s= \begin{cases}-\frac{\mathrm{e}^{r^{\prime}-(p+2) r} r}{p+2}+\frac{\mathrm{e}^{r^{\prime}}-\mathrm{e}^{r^{\prime}-(p+2) r}}{(p+2)^{2}}, & r^{\prime} \leq 0, \\
\frac{1}{(p+1)^{2}}-\frac{\mathrm{e}^{-(p+1) r^{\prime}}(2 p+3)}{(p+1)^{2}(p+2)^{2}}-\frac{\mathrm{e}^{-(p+1) r^{\prime}} r^{\prime}}{(p+1)(p+2)} & \\
-\frac{\mathrm{e}^{r^{\prime}-(p+2) r}}{(p+2)^{2}}-\frac{\mathrm{e}^{r^{\prime}}-(p+2) r}{p+2}, & 0<r^{\prime} \leq r, \\
-\frac{\mathrm{e}^{-(p+1) r} r}{p+1}+\frac{1-\mathrm{e}^{-(p+1) r}}{(p+1)^{2}}, & r^{\prime}>r,\end{cases} \\
& \int_{0}^{r} K\left(r^{\prime}, 2\right) \mathrm{e}^{-p s} \mathrm{~d} s= \begin{cases}\frac{\mathrm{e}^{r^{\prime}}-\mathrm{e}^{r^{\prime}-(p+2) r}}{p+2}, & r^{\prime} \leq 0, \\
\frac{1}{p+1}-\frac{\mathrm{e}^{r^{\prime}-(p+2) r}}{p+2}-\frac{\mathrm{e}^{-(p+1) r^{\prime}}}{(p+1)(p+2)}, & 0<r^{\prime} \leq r, \\
\frac{1-\mathrm{e}^{-(p+1) r}}{p+1}, & r^{\prime}>r,\end{cases} \\
& \int_{r}^{\infty} K\left(r^{\prime}, 2\right) \mathrm{e}^{-p s} \mathrm{~d} s= \begin{cases}\frac{\mathrm{e}^{r^{\prime}-(p+2) r}}{p+2}, & r^{\prime} \leq r, \\
\frac{\mathrm{e}^{-(p+1) r}}{p+1}-\frac{\mathrm{e}^{-(p+1) r^{\prime}}}{(p+1)(p+2)}, & r^{\prime}>r .\end{cases}
\end{aligned}
$$

This implies that, for $r^{\prime} \leq 0$, the function $K^{n+1}$ is given by

$$
K^{n+1}\left(r^{\prime}, r\right)=\sum_{p, q=0}^{n} \tilde{a}_{p, q}^{n+1} \mathrm{e}^{p r^{\prime}-(q+2) r},
$$

where

$$
\begin{aligned}
& \tilde{a}_{p, q}^{n+1}=\delta_{p, 0} \sum_{k=0}^{n} \frac{a_{k, q}^{n}}{k+1}-\Theta_{p, 1} \frac{a_{p-1, q}^{n}}{p(p+1)} \\
&+\delta_{p, 1}\left[\delta_{q, n-1} \frac{(-1)^{n-1}}{(n-1)^{!}}-\delta_{q, n} \frac{(-1)^{n-1}}{(n-1)^{!}}+\tilde{\Theta}_{q, n-2} \frac{(-1)^{n}}{(n-q-2)^{!}(q)^{!}(n-q)^{2}}\right. \\
& \\
&-\delta_{q, n} \sum_{k=0}^{n-2} \frac{(-1)^{n}}{(k)^{!}(n-k-2)^{!}(p+2)^{2}}+\sum_{k=0}^{n} \frac{b_{k, q}^{n}}{k+2}-\sum_{k=0}^{q-2} \frac{b_{k, q-k-2}^{n}}{k+2} \\
&\left.+\sum_{k=0}^{q-2} \frac{c_{k, q-k-2}^{n}}{k+2}+\delta_{q, n} \frac{(-1)^{n-1}}{(n-1)^{!}(n+1)}\right] .
\end{aligned}
$$


By Lemma $6, \tilde{a}_{p, q}^{n+1}$ is equal to $a_{p, q}^{n+1}$. Similarly, for $0<r^{\prime} \leq r$, the function $K^{n+1}$ takes the form

$$
\begin{aligned}
K^{n+1}\left(r^{\prime}, r\right)= & \sum_{q=0}^{n} \beta_{q}^{n+1} \mathrm{e}^{r^{\prime}-(q+2) r}+\sum_{p=0}^{n-1} \frac{(-1)^{n+1} r^{\prime} \mathrm{e}^{-p\left(r^{\prime}-r\right)-(n+1) r}}{(p)^{!}(n-p-1)^{!}} \\
& +\sum_{p=0}^{n+1} \sum_{q=0}^{n+1} \tilde{b}_{p, q}^{n+1} \mathrm{e}^{-p r^{\prime}-(q+2) r},
\end{aligned}
$$

where

$$
\begin{aligned}
\beta_{q}^{n+1}= & \delta_{q, n}\left[\frac{(-1)^{n-1}}{(n-1)^{!}(n+1)}-\frac{(-1)^{n-1}}{(n-1)^{!}}-\sum_{k=0}^{n-2} \frac{(-1)^{n}}{(k)^{!}(n-k-2)^{!}(k+2)^{2}}\right] \\
& -\sum_{k=0}^{q-2} \frac{b_{k, q-k-2}^{n}}{k+2}+\sum_{k=0}^{q-2} \frac{c_{k, q-k-2}^{n}}{k+2}
\end{aligned}
$$

and

$$
\begin{aligned}
\tilde{b}_{p, q}^{n+1}= & \delta_{p, 1} \sum_{k=0}^{n} \frac{a_{k, q}^{n}}{k+2}-\Theta_{p, 1} \delta_{q, n-p-1} \frac{(-1)^{n}(2 p+1)}{(p-1)^{!}(n-p-1)^{!} p^{2}(p+1)^{2}} \\
& +\delta_{p, 0}\left[\delta_{q, n-1} \frac{(-1)^{n-1}}{(n-1)^{!}}+\tilde{\Theta}_{q, n-2} \frac{(-1)^{n}}{(n-q-2)^{!}(q)^{!}(n-q-1)^{2}}+\sum_{k=0}^{n} \frac{b_{k, q}^{n}}{k+1}\right] .
\end{aligned}
$$

Lemma 5 implies that $\beta_{q}^{n+1}=\delta_{q, n}(-1)^{n} /(n)^{!}$, and, by Lemma $7, \tilde{b}_{p, q}^{n+1}$ is equal to $b_{p, q}^{n+1}$. Finally, for $r^{\prime}>r$, the function $K^{n+1}$ becomes

$$
K^{n+1}\left(r^{\prime}, r\right)=\frac{(-1)^{n} \mathrm{e}^{-n r^{\prime}-r}}{(n)^{!}}+\sum_{p=0}^{n-1} \frac{(-1)^{n+1} r \mathrm{e}^{-p\left(r^{\prime}-r\right)-(n+1) r}}{(p)^{!}(n-p-1)^{!}}+\sum_{p=0}^{n+1} \sum_{q=0}^{n+1} \tilde{c}_{p, q}^{n+1} \mathrm{e}^{-p r^{\prime}-(q+2) r},
$$

where

$$
\begin{aligned}
\tilde{c}_{p, q}^{n+1}= & \delta_{p, 1} \sum_{k=0}^{n} \frac{a_{k, q}^{n}}{k+2}-\Theta_{p, 1} \frac{c_{p-1, q}^{n}}{p(p+1)} \\
+\delta_{p, 0}\left[\tilde{\Theta}_{q, n-2} \frac{(-1)^{n}}{(n-q-2)^{!}(q)^{!}(n-q-1)^{2}}\right. & \\
& +\delta_{q, n-1} \sum_{k=0}^{n-2} \frac{(-1)^{n}}{(k)^{!}(n-k-2)^{!}(k+1)^{2}}+\sum_{k=0}^{n} \frac{b_{k, q}^{n}}{k+1}-\sum_{k=0}^{q-1} \frac{b_{k, q-k-1}^{n}}{k+1} \\
& \left.-\delta_{q . n-1} \frac{(-1)^{n-1}}{n !^{2}}+\sum_{k=0}^{q-1} \frac{c_{k, q-k-1}^{n}}{k+1}\right] .
\end{aligned}
$$


In Lemma 8 it was shown that $\tilde{c}_{p, q}^{n+1}$ equals $c_{p, q}^{n+1}$. Combining (23), (25), and (27) proves the theorem because it follows that, for $r \geq 0$, the values $K^{n+1}\left(r^{\prime}, r\right)$ are given by

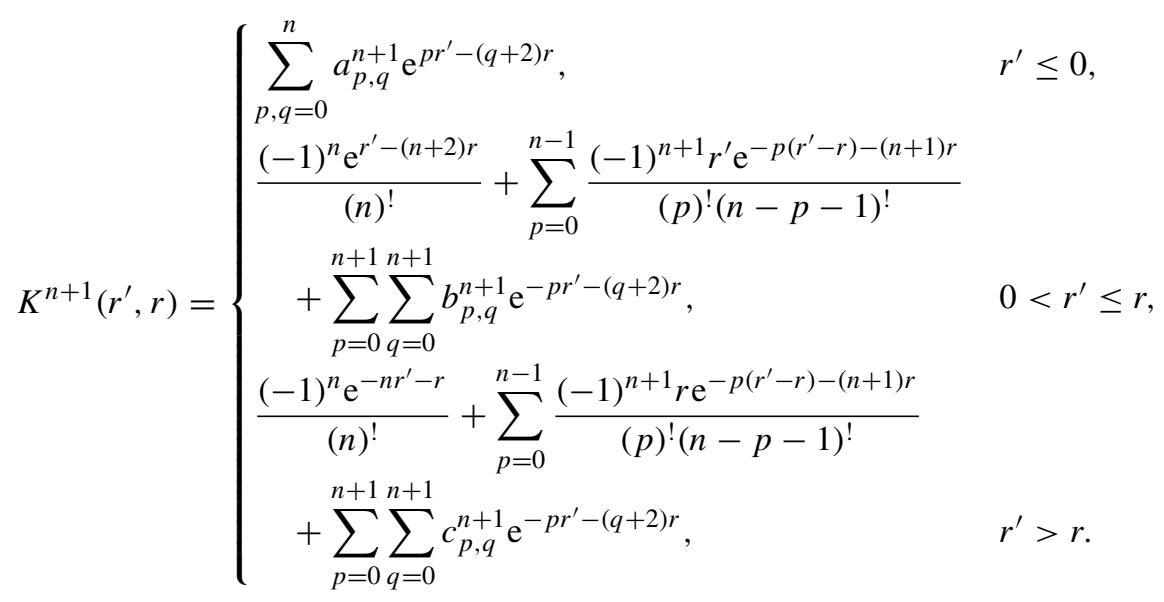

\section{Discussion}

The way in which Theorem 2 was proved gives little insight into how we arrive at expressions (7)-(9) for the generating functions $A_{p, q}, B_{p, q}$, and $C_{p, q}$ in the first place. It appears pertinent to briefly comment on how we derived these formulae. The first step was to compute the kernels $K^{n}$ for low values of $n$ from the Chapman-Kolmogorov equation (22) and to observe that they have the form asserted in Theorem 2. In the next step we guessed the expression for the part of $K^{n}\left(r^{\prime}, r\right)$ not involving the coefficients $a_{p, q}^{n}, b_{p, q}^{n}$, and $c_{p, q}^{n}$ so that the problem was reduced to solving the recurrence equations (24), (26), and (28). Assuming the validity of Lemma 5, it turns out that the first two of these recurrence equations can be relatively easily solved first for $G(z)$, which is, up to the factor $(-z)^{q} /(q)^{!}$, the generating function of $\left(a_{1, q}^{n}+b_{1, q}^{n}\right)_{n \geq 1}$, then for $A_{p, q}$, and, finally, for $B_{p, q}$. The third recursion for $\left(c_{p, q}^{n}\right)$ was simplified by the empirical observation that

$$
\sum_{k=0}^{q-1} \frac{b_{k, q-k-1}^{n}}{k+1}-\sum_{k=0}^{q-1} \frac{c_{k, q-k-1}^{n}}{k+1}=\delta_{q, n+1}\left[\frac{(-1)^{n-1}}{n !^{2}}+\sum_{k=0}^{q-1} \frac{(-1)^{q}}{(k)^{!}(q-k-1)^{!}(k+1)^{2}}-d_{q}\right]
$$

for some real numbers $d_{q}, q \geq 0$, and then solved for $C_{p, q}$. The educated guesses made in the course of this derivation are justified ex posteriori by the proofs presented in this paper.

Our original motivation was to derive an explicit expression for the asymptotic variance (5). For this purpose, knowledge of the generating function of the coefficients of the $n$-step transition kernel, as opposed to knowledge of the coefficients themselves, is sufficient. In order to evaluate the infinite sum appearing in (5), one is primarily interested in sums of the form $\sum_{n \geq 1} a_{p, q}^{n}$, which is equal to $A_{p, q}(1)$, provided that this number is finite. Carrying out the computations, however, turns out to be quite subtle and the results will be reported elsewhere.

It is natural to ask whether the results presented in this paper can be extended to the first passage percolation problem on $\mathbb{N} \times\{0,1, \ldots, k\}, k \geq 2$. Conceptually, our approach carries over to this setting only if we consider semidirected percolation in which the horizontal edges may be traversed in only one direction; the combinatorics involved in computing the one-step transition kernel of the Markov chain $\Delta$ as well as the explicit iteration of the ChapmanKolmogorov equation (22), however, soon become unmanageable for larger values of $k$. For 
the undirected first passage percolation problem, there is the possibility that the shortest path $\left\{(0,0)=p_{0}, p_{1}, \ldots, p_{N-1}, p_{N}=(n, 0)\right\}, p_{i}=\left(x_{i}, y_{i}\right)$, between $(0,0)$ and $(n, 0)$ backtraces, by which we mean that there exist indices $0 \leq i<j \leq N$ such that $x_{j}<x_{i}$. The possible occurrence of such configurations prevents an extension of our recursive method to broader graphs in the undirected setting. One might also wonder if similar results can be obtained for more a general class of edge-weight distributions P. It is easy to see that the Markov property of $\Delta$ does not depend on the choice of $\mathrm{P}$ and an analysis of our proofs shows that the validity of the central limit theorem (Theorem 1) as well as expression (5) for the asymptotic variance is not affected by choosing a different edge-weight distribution either, provided that we can prove that the stationary distribution $\tilde{\pi}$ and the one-step kernel $K$ satisfy the moment and mixing conditions used in the proof of Theorem 1 . It is, however, very difficult to evaluate the formula for the $n$-step transition kernel explicitly, if $\mathrm{P}$ is not the exponential distribution, although our approach via generating functions remains likewise applicable.

\section{Acknowledgements}

This work was completed during a visit to the Statistics Department of Colorado State University, whose hospitality the author gratefully acknowledges. The author also gratefully acknowledges financial support from the Technische Universität München Institute for Advanced Study funded by the German Excellence Initiative and from the International Graduate School of Science and Engineering.

\section{References}

[1] Abramowitz, M. and Stegun, I. A. (eds) (1992). Handbook of Mathematical Functions with Formulas, Graphs, and Mathematical Tables. Dover, New York.

[2] Ahlberg, D. (2009). Asymptotics of first-passage percolation on 1-dimensional graphs. Preprint. Department of Mathematical Sciences, Chalmers University of Technology and Göteborg University.

[3] Aldous, D., Lovász, L. AND Winkler, P. (1997). Mixing times for uniformly ergodic Markov chains. Stoch. Process. Appl. 71, 165-185.

[4] Altmann, M. (1993). Reinterpreting network measures for models of disease transmission. Social Networks 15, 1-17.

[5] Bhamidi, S., VAn der Hofstad, R. And Hooghiemstra, G. (2010). First passage percolation on random graphs with finite mean degrees. Ann. Appl. Prob. 20, 1907-1965.

[6] Chatterjee, S. And Dey, P. S. (2009). Central limit theorem for first-passage percolation time across thin cylinders. Preprint. Available at http://arxiv.org/abs/0911.5702v2.

[7] Chen, X. (1999). Limit theorems for functionals of ergodic Markov chains with general state space. Mem. Amer. Math. Soc. 139, 203 pp.

[8] Flaxman, A., Gamarnik, D. and Sorkin, G. (2006). First-passage percolation on a width-2 strip and the path cost in a VCG auction. In Internet and Network Economics (Lecture Notes Comput. Sci. 4286), Springer, Berlin, pp. 99-111.

[9] Graham, R. L., Grötschel, M. and Lovász, L. (eds) (1995). Handbook of Combinatorics, Vol. 2. NorthHolland, Amsterdam.

[10] Grimmett, G. and Kesten, H. (1984). First-passage percolation, network flows and electrical resistances. Z. Wahrscheinlichkeitsth. 66, 335-366.

[11] Hammersley, J. M. and Welsh, D. J. A. (1965). First-passage percolation, subadditive processes, stochastic networks, and generalized renewal theory. In Bernouli, Bayes, Laplace. Anniversary Volume, Springer, New York, pp. 61-110.

[12] Kesten, H. (1987). Percolation theory and first-passage percolation. Ann. Prob. 15, 1231-1271.

[13] Nummelin, E. And Tuominen, P. (1982). Geometric ergodicity of Harris recurrent Markov chains with applications to renewal theory. Stoch. Process. Appl. 12, 187-202.

[14] Renlund, H. (2010). First-passage percolation with exponential times on a ladder. Combinatorics Prob. Comput. 19, 593-601.

[15] Schlemm, E. (2009). First-passage percolation rates on width-two stretches with exponential link weights. Electron. Commun. Prob. 140, 424-434. 
[16] SeppäLÄINEN, T. (1998). Exact limiting shape for a simplified model of first-passage percolation on the plane. Ann. Prob. 26, 1232-1250.

[17] Smythe, R. T. and Wierman, J. C. (1978). First-Passage Percolation on the Square Lattice (Lecture Notes Math. 671). Springer, Berlin.

[18] Sood, V., Redner, S. And ben Avraham, D. (2005). First-passage properties of the Erdős-Renyi random graph. J. Phys. A 38, 109-123.

[19] Van der Hofstad, R., Hooghiemstra, G. and Van Mieghem, P. (2001). First-passage percolation on the random graph. Prob. Eng. Inf. Sci. 15, 225-237.

[20] WiLf, H. S. (2006). Generatingfunctionology, 3rd edn. A K Peters, Wellesley, MA. 